\title{
Chapter 19 \\ Appendix D: Defining Boolean and Fuzzy \\ Logic Operators
}

\author{
Val Lowndes
}

\subsection{Definition Boolean Logic}

If an element $x$

is contained in set $A$ then

$\mu_{A}(x)=1 \quad$ if $\quad x \in A$

is not contained in set $A$ then

As

$\mu_{A}(x)=0$

and

$\mu_{A \cup B}(x)=1 \quad$ if $\quad x \in A$ or $x \in B$

Then it follows that

$\mu_{A \cap B}(x)=1 \quad$ if $\quad x \in A$ and $x \in B$

and

$A \cup B \rightarrow \mu_{A \cup B}(x)=\max \left[\mu_{A}(x), \mu_{B}(x)\right]$

$A \cap B \rightarrow \mu_{A \cap B}(x)=\min \left[\mu_{A}(x), \mu_{B}(x)\right]$

\subsection{Definition Fuzzy Logic}

If an element $x$

has some membership in set $A$ then

has no membership in set $A$ then

$$
\begin{aligned}
& \mu_{A}(x)=k_{A} \quad 0<k_{A} \leq 1 \\
& \mu_{A}(x)=0 \\
& A \cup B \rightarrow \mu_{A \cup B}(x)=\max \left[\mu_{A}(x), \mu_{B}(x)\right] \\
& A \cap B \rightarrow \mu_{A \cap B}(x)=\min \left[\mu_{A}(x), \mu_{B}(x)\right]
\end{aligned}
$$

define

and

thus

$$
0 \leq \mu_{A \cup B}(x) \leq 1 \quad \text { and } \quad 0 \leq \mu_{A \cap B}(x) \leq 1
$$

V. Lowndes $(\bowtie)$

University of Derby, Kedleston Road, DE22 1GB Derby, UK

e-mail: V.P.Lowndes@derby.ac.uk

S. Berry et al. (eds.), Guide to Computational Modelling for Decision Processes, Simulation Foundations, Methods and Applications, 